\title{
Clinically Amyopathic Dermatomyositis Associated with Recurrence of Esophageal Cancer: A Case Report
}

\author{
Anna Eversman, $\mathrm{BS}^{1}$, Sayeeda Ahsanuddin, $\mathrm{MD}^{2}$, Joel Saltzman, $\mathrm{MD}^{1,3}$, Paula Silverman, \\ $\mathrm{MD}^{1,3}$, Mara G Beveridge, $\mathrm{MD}^{1,2}$ \\ ${ }^{1}$ Case Western Reserve University School of Medicine, Cleveland, $\mathrm{OH}$ \\ ${ }^{2}$ University Hospitals Cleveland Medical Center, Department of Dermatology, Cleveland, $\mathrm{OH}$ \\ ${ }^{3}$ University Hospitals Cleveland Medical Center, Department of Medical Oncology, Cleveland, $\mathrm{OH}$
}

\section{ABSTRACT}

Background: Approximately $20 \%$ of patients displaying skin changes characteristic of dermatomyositis do not develop clinical or enzymatic evidence of muscle inflammation within 6 months of onset, leading to a diagnosis of clinically amyopathic dermatomyositis (CADM). Current literature suggests the incidence of malignancy is lower in CADM patients compared to patients with dermatomyositis. It is still necessary for clinicians to screen for malignancy in CADM patients, however, as data on CADM remains limited.

Case Presentation: We report a case of new-onset CADM associated with recurrent esophageal adenocarcinoma for clinical interest and to add to the limited literature on CADM.

Conclusion: To date, there is only one report of concurrent CADM and esophageal cancer. We present this case for clinical interest and to add to the limited literature on CADM.

\section{INTRODUCTION}

Dermatomyositis is a rare inflammatory disorder characterized by pathognomonic skin changes and muscle weakness. Classic cutaneous findings include: the heliotrope rash, Gottron's papules, and poikilodermatous patches. Approximately $20 \%$ of patients displaying characteristic skin changes do not develop clinical or enzymatic evidence of muscle inflammation within 6 months of onset, leading to a diagnosis of clinically amyopathic dermatomyositis (CADM) ${ }^{1} \quad$ Muscle weakness rarely occurs after a prolonged delay, suggesting CADM may be a clinical entity distinct from dermatomyositis. ${ }^{2}$ Current literature suggests the incidence of malignancy is lower in CADM patients compared to patients with dermatomyositis. ${ }^{3}$ It is still necessary for clinicians to screen for malignancy in CADM patients, however, as data on CADM remains limited. We report a case of new-onset CADM associated with recurrent esophageal adenocarcinoma.

\section{CASE PRESENTATION}

A 64-year-old female with a history of stage III esophageal cancer presented in June of 2019 with a one-month history of a pink, pruritic rash involving the face, upper chest, and extremities (Figure 1). She denied muscle tenderness or weakness. Her esophageal cancer was diagnosed in November of 2018 and was treated with neoadjuvant carboplatin, paclitaxel and concurrent radiation followed by an

May 2021 Volume 5 Issue 3 


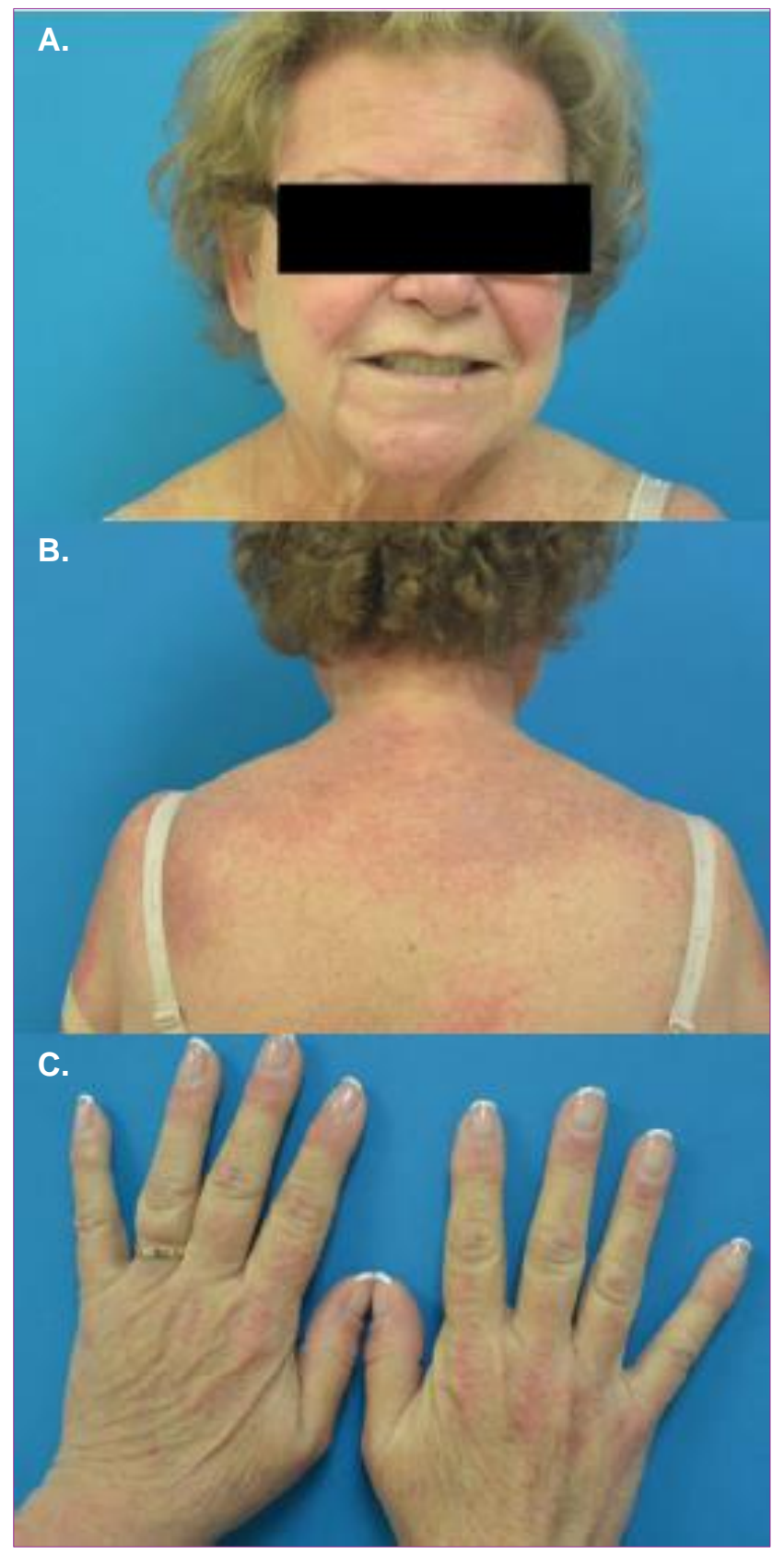

Figure 1. (A) Erythema of the face in a butterfly distribution, (B) poikilodermatous patches on the upper back in a shawl distribution, (C) and pink papules covering the dorsal finger joints.

esophagectomy. Her past cancer history was also notable for invasive ductal carcinoma of the right breast, diagnosed in January of 2017 and treated with lumpectomy, radiation, anastrozole, and exemestane. She also had multiple colon polyps. Of note, the patient had a PET/CT scan four months prior to presentation, which showed no evidence of metastatic disease.

Physical exam was remarkable for confluent, bright pink patches and plaques on the extensor surface of the upper extremities bilaterally, with perifollicular papules at periphery. A few scattered, pink perifollicular papules were also seen on the thighs. Pink, poikilodermatous patches were present in a shawl distribution on the chest and upper back. Erythema of the face was present in a butterfly distribution, and pink papules covered the dorsal finger joints.

Laboratory findings were notable for positive anti-nuclear antibodies (ANA) in a homogenous pattern. An ENA panel was negative. Creatine kinase and aldolase levels were within normal limits. A punch biopsy of the left upper extremity demonstrated basal layer vacuolization with occasional dyskeratosis and a mild superficial lymphocytic infiltrate (Figure 2). A sample obtained for direct immunofluorescence was nonreactive.

A repeat PET/CT showed hypermetabolic nodular soft tissue thickening at prior surgical sites, as well as two ill-defined hypoechoic lesions with increased FDG uptake in the liver. Hypermetabolic mediastinal, celiac, and right internal mammary lymph nodes were also noted, raising concern for residual or recurrent esophageal carcinoma versus breast cancer.

Expedited oncologic surveillance was discussed with her care team following diagnosis of CADM. Biopsies were performed on concerning liver lesions identified by her recent PET scan, and pathology revealed metastatic esophageal adenocarcinoma. The patient's cutaneous symptoms have improved with an oral 


\section{SKIN}

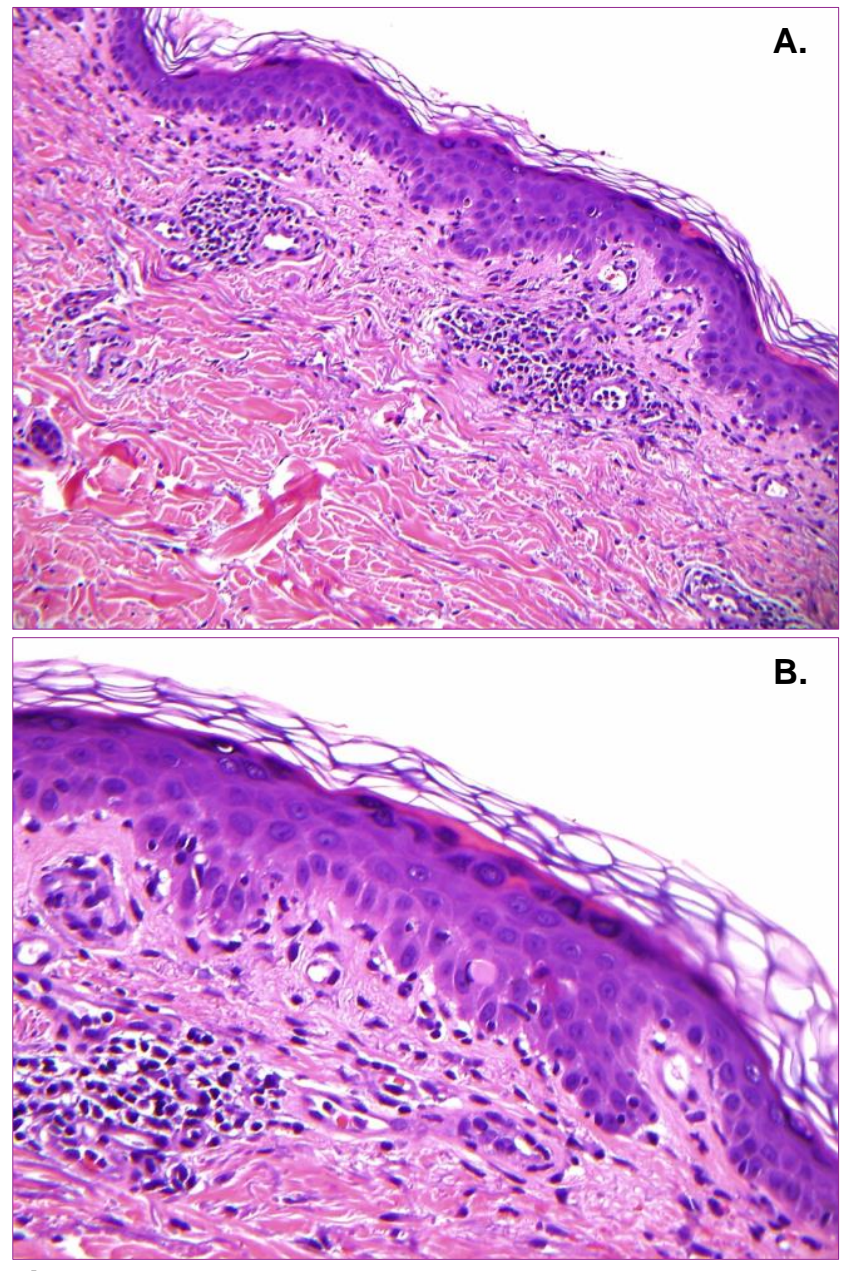

Figure 2: Histopathologic examination revealed basal layer vacuolization with occasional dyskeratosis and a mild superficial lymphocytic infiltrate (A, H\&E 40x) (B, H\&E 20x).

prednisone taper, hydroxychloroquine, and topical steroids. Treatment of her recurrent esophageal cancer with FOLFOX chemotherapy (folinic acid, 5-fluorouracil, and oxaliplatin) is scheduled to begin this month.

\section{DISCUSSION}

To our knowledge, this is the first report of CADM associated with recurrence of esophageal cancer. Exacerbations of classic dermatomyositis in the setting of malignancy recurrence are common, however there are few reports of new-onset CADM associated with malignancy recurrence. ${ }^{4}$ Additionally, esophageal cancer is not frequently linked to the inflammatory myopathies. ${ }^{5}$ To date, there is only one report of concurrent CADM and esophageal cancer. ${ }^{5}$ We present this case for clinical interest and to add to the limited literature on CADM.

Dermatomyositis, an inflammatory myopathy, encompasses a spectrum of presentations with variable skin and muscle involvement. As skin changes commonly precede muscle involvement, the diagnosis of CADM is reserved for patients with no clinical or enzymatic evidence of muscle involvement within 6 months of onset. ${ }^{6}$ CADM refers to both amyopathic disease and cases with subclinical myositis on biopsy, termed hypomyopathic disease. ${ }^{7}$ The unique clinical presentation of CADM as well as differences in associated antibodies suggest independent analysis of this subset of patients is warranted. ${ }^{2}$

Between 10 and $50 \%$ of patients with dermatomyositis have an underlying neoplasm, with cancer of the ovaries, breast, lung, colon, cervix, thyroid, and bladder reported most frequently. ${ }^{6,7}$ Studies on the malignancy risk conferred by CADM have yielded conflicting results. Early reports suggested there was no correlation between CADM and malignancy. ${ }^{8}$ More recent literature, however, revealed CADM and dermatomyositis are associated with an equal age-dependent risk of malignancy. ${ }^{6,7}$ Carcinomas of the nasopharynx, breast, ovary, and lung are commonly reported in patients with CADM. $^{6}$ Patients with autoantibodies in addition to ANA are less likely to develop a malignancy, suggesting these antibodies are correlated with true autoimmune etiologies. ${ }^{7}$

As malignancies may be diagnosed before, during, or after the onset of May 2021 Volume 5 Issue 3 
dermatomyositis, the temporal relationship between these two conditions is unclear. Resolution of dermatomyositis has been reported following tumor resection, suggesting the inflammatory myopathy represents an immune response to the cancer. $^{9}$ It is possible, however, that immunosuppression secondary to dermatomyositis treatment increases the risk of malignancy. ${ }^{10}$ Increased testing and surveillance following the diagnosis of dermatomyositis may also be a contributing factor. Independent of the etiology, physicians should be aware of malignancies correlated to inflammatory myopathies to ensure patients undergo appropriate screening.

As dermatomyositis and CADM are associated with internal malignancies, additional workup is warranted in both populations. A thorough history, physical examination, and baseline laboratory testing is recommended for all patients with newonset dermatomyositis. ${ }^{11}$ Patients should also be up-to-date on all age- and genderappropriate cancer screening. Although further testing may be obtained based on patient history, there are currently no consensus guidelines. Imaging is often reserved for patients with additional findings concerning for malignancy. ${ }^{11}$ As the majority of dermatomyositis patients harboring internal malignancies are otherwise asymptomatic, more liberal use of imaging may be beneficial. ${ }^{10}$ In a study from Leatham et al, malignancies in patients with dermatomyositis were most frequently detected by CT scan. As most malignancies reveal themselves within 5 years of the onset of cutaneous eruption, repeat testing during this interval may also be of utility. ${ }^{7}$

\section{Conflict of Interest Disclosures: None}

Funding: None
Corresponding Author:

Mara G Beveridge, MD

11100 Euclid Ave

Lakeside 3500

Cleveland, $\mathrm{OH} 44106$

Phone: 216-514-8630

Email: Mara.Beveridge@uhhospitals.org

\section{References:}

1. Pinard J, Femia AN, Roman M, et al. Systemic Treatment for Clinically Amyopathic Dermatomyostitis at 4 Tertiary Care Centers. JAMA Dermatol. 2019;155(4):494-496.

2. Sato S, Kuwana M. Clinically amyopathic dermatomyositis. Curr Opin Rheumatol 2010;22:63943.

3. Bowerman K, Pearson DR, Okawa J, Werth VP. Malignancy in dermatomyositis: A retrospective study of 201 patients seen at the University of Pennsylvania. J Am Acad Dermatol. 2020 Jul;83:117-122.

4. Goyal S, Nousari HC. Paraneoplastic amyopathic dermatomyositis associated with breast cancer recurrence. J Am Acad Dermatol.1999;(41):874-875.

5. Kikuchi K, Seto Y, Matsubara T, et al. Amyopathic dermatomyositis associated with esophageal cancer. Int J Dermatol. 2008;47:310-311.

6. Gerami P, Schope JM, Mcdonald L, Walling HW, Sontheimer RD. A systematic review of adult-onset clinically amyopathic dermatomyositis (dermatomyositis siné myositis): a missing link within the spectrum of the idiopathic inflammatory myopathies. J Am Acad Dermatol. 2006;54(4):597613.

7. Galimberti F, Li Y, Fernandez AP. Clinically amyopathic dermatomyositis: clinical features, response to medications and malignancy-associated risk factors in a specific tertiary-care-centre cohort. $\mathrm{Br}$ J Dermatol. 2016;174(1):158-64.

8. Yu, B. A clinical analysis of cutaneous type dermatomyositis. Zhongguo Yi Xue Ke Xue Yuan Xue Bao. 1994;16:394-396.

9. Joseph CG, Darrah E, Shah AA, et al. Association of the autoimmune disease scleroderma with an immunologic response to cancer. Science. 2014;343:152-7.

10. Leatham H, Schadt $\mathrm{C}$, Chisolm S, et al. Evidence supports blind screening for internal malignancy in dermatomyositis: Data from 2 large US dermatology cohorts. Medicine (Baltimore). 2018;97(2):e9639.

11. Sontheimer RD. Clinically amyopathic dermatomyositis: what can we now tell our patients? Arch Dermatol. 2010;146(1):76-80. 